\title{
Uni-Port Total Thoracoscopic Surgery Versus Median Sternotomy for Redo Tricuspid Valve Replacement: A Retrospective Study
}

\author{
Licheng Yan, Fuzhen Zheng, Haiyu Chen, MD, Jiayin Bao, Guoxing Weng, MD \\ Department of Cardiovascular Surgery, Fujian Provincial Hospital, Fuzhou, Fujian, P.R.China
}

\section{ABSTRACT}

Background: This study compared the perioperative and follow-up period data of patients who underwent redo tricuspid valve replacements performed via thoracoscopic surgery or median sternotomy. The purpose was to evaluate the feasibility, safety, and surgical outcomes of redo tricuspid valve replacement via uni-port thoracoscopic surgery.

Methods: Forty-nine patients with severe tricuspid valve regurgitation after left-side valve replacement underwent redo tricuspid valve replacements in our hospital from April 2012 to September 2019. Twenty-six patients underwent uniport total thoracoscopy surgery, whereas 23 patients had the surgery performed via median sternotomy. We collected perioperative and 3- to 36-month postoperative data.

Results: No deaths occurred in the intraoperative period. Time of cardiopulmonary bypass in the study group significantly was longer than that in the control group $(P<.05)$, but the operative times in the study and control groups were not significantly different. Thoracic drainage, length of ICU stay, postoperative hospital stay, and complication rates in the study group were significantly different from those in the control group $(P<.05)$. Throughout the follow-up period, uni-port total thoracoscopic TVR was not inferior to traditional surgery with respect to cardiac function and recurrence of tricuspid valve regurgitation.

Conclusions: Uni-port total thoracoscopic tricuspid valve replacement is safe, feasible and effective, and that can be considered as a primary treatment strategy for patients with severe TR after previous left-sided valve procedure.

\section{INTRODUCTION}

Secondary tricuspid valve regurgitation (TR) after a left-sided valve procedure is gaining increasing attention, although it extensively has been misunderstood. Secondary TR is a progressive disorder characterized by a spiral of right ventricular chamber enlargement leading to further annular dilatation and more tricuspid regurgitation [El-Eshmawi 2018; Pinney 2012]. Nevertheless, surgical tricuspid surgery

Received March 13, 2020; accepted March 18, 2020.

Correspondence: Guoxing Weng, MD, Fujian Provincial Hospital, 134 East St, Fuzhou, Fujian 350001, P.R.China; 0086-13905907279; fax: 008613905907279 (e-mail: gxw001@sina.com). has been avoided for years because of the misleading concept that tricuspid regurgitation should disappear once the primary left-sided problem has been eliminated. Instead, during the last decade, many investigators have reported evidence in favor of a more aggressive surgical approach to functional tricuspid regurgitation, recognizing the risk of progressive tricuspid insufficiency in patients with moderate or lesser degrees of tricuspid regurgitation and tricuspid annular dilatation. Furthermore, severe tricuspid insufficiency occurred in $25 \% \sim 75 \%$ of the patients after the left-sided procedures [Tornos 2015; Bellavia 2014; Dreyfus 2005; Van de Veire 2011; Nishimura 2014; He 2012; Pozzoli 2016; Lange 2017]. There has been increasing evidence showing that TR could
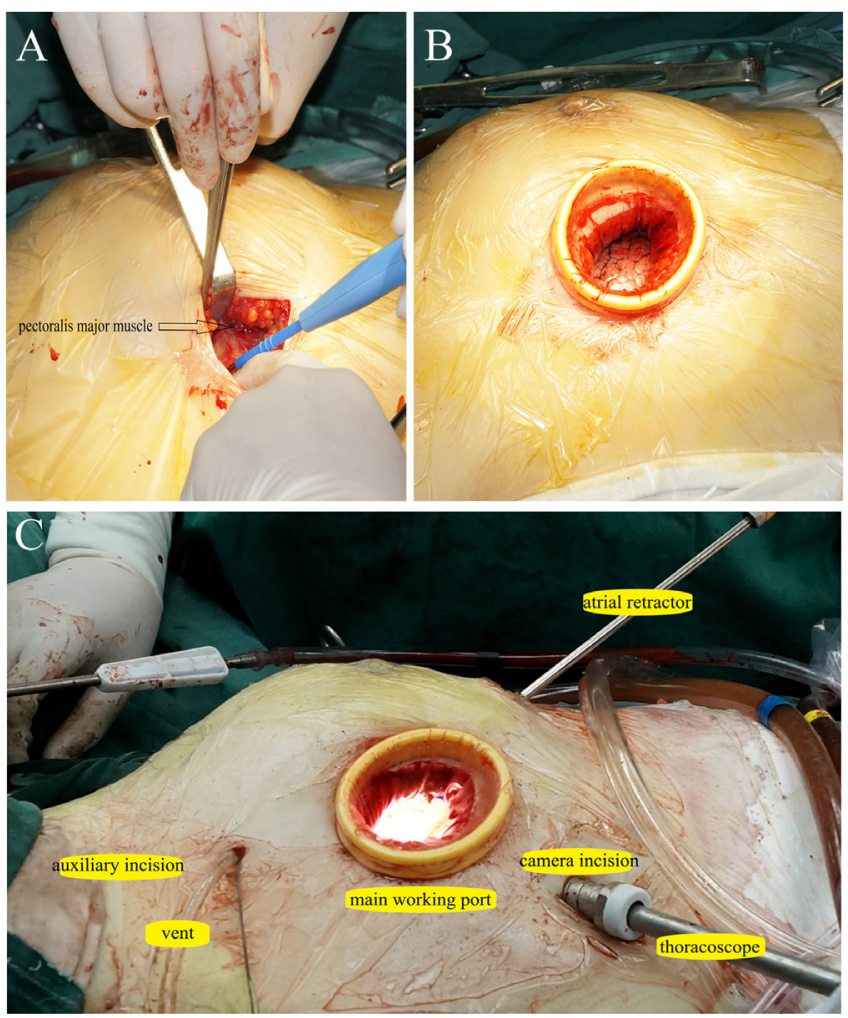

Figure 1. The main working port was established, all surgical instruments were set-up for surgery. A, B, Dissecting a space beneath the pectoralis major muscle, thoracotomy was performed in the fourth intercostal space for the main working port, soft-tissue retractors were used. C, Complete set-up for uni-port total thoracoscopic TVR. 
Table 1. Clinical characteristics of the patients

\begin{tabular}{lccc}
\hline & Study group $(\mathrm{N}=26)$ & Control group $(\mathrm{N}=23)$ \\
\hline Age (years) & $56.3 \pm 6.5$ & $53.0 \pm 6.6$ & .093 \\
Sex (male/female\%) & $23.8 \%(5 / 21)$ & $21.1 \%(4 / 19)$ & 1.000 \\
$\mathrm{BMI}\left(\mathrm{kg} / \mathrm{m}^{2}\right)$ & $22.0 \pm 3.3$ & $23.3 \pm 2.5$ & .130 \\
The interval between the two operations & $16.2 \pm 5.2$ & $16.4 \pm 7.3$ \\
Hypertension (\%) & $46.2 \%(12)$ & $34.8 \%(8)$ & .877 \\
Diabetes (\%) & $19.2 \%(5)$ & $8.7 \%(2)$ \\
Atrial fibrillation (\%) & $96.2 \%(25)$ & $82.6 \%(19)$ \\
Congestive liver failure or hepatic insufficiency (\%) & $21.7 \%(5)$ \\
Oliguria or renal dysfunction (\%) & $3.8 \%(1)$ & $8.7 \%(2)$ \\
Hydrothorax or ascites (\%) & $26.9 \%(7)$ & $13.0 \%(3)$ \\
NYHA function class II (\%) & $26.9 \%(7)$ & $17.4 \%(4)$ \\
NYHA function class III (\%) & $65.4 \%(17)$ & $66.6 \%(15)$ \\
NYHA function class IV (\%) & $7.7 \%(2)$ & $17.4 \%(4)$ \\
\hline
\end{tabular}

BMI: body mass index; NYHA: New York Heart Association

Table 2. Preoperative demographic characteristics

\begin{tabular}{|c|c|c|c|c|c|}
\hline \multicolumn{6}{|l|}{ Severities of tricuspid regurgitation } \\
\hline ++++ & 22 & 84.6 & 17 & 73.9 & .483 \\
\hline LVEF (\%) & $57.7 \pm 2.1$ & $58.1 \pm 5.0$ & 0.742 & & \\
\hline Internal diameters of RV (mm) & $48.3 \pm 8.9$ & $47.7 \pm 9.2$ & 0.823 & & \\
\hline Internal diameters of LA (mm) & $54.9 \pm 10.2$ & $53.6 \pm 6.9$ & 0.610 & & \\
\hline $\mathrm{PAP}(\mathrm{mmHg})$ & $45.4 \pm 7.2$ & $44.7 \pm 16.6$ & 0.846 & & \\
\hline
\end{tabular}

LA, left atrium; LVEF, left ventricular ejection fraction; PAP, pulmonary artery pressure; RA, right atrium; RV, right ventricle

be persistent after a left-sided valve procedure, and this is associated with substantial reduction in survival and quality of life. Patients who have moderate to severe TR should be considered for surgical intervention; the aggressive strategy was safe and effective and associated with improved long-term right-sided cardiac remodeling [El-Eshmawi 2018; Benedetto 2012; Gosev 2016; Jamieson 1999; Anyanwu 2010; Chikwe 2015]. However, cardiac pump function, surgical endurance, and organ function status should be considered. Once TR is severe and right-sided heart failure sets in, a reoperation on the TV after left-sided valve surgery carries an operative mortality risk of $10 \%-30 \%$ [Nishimura 2014; McCarthy 2004; Pfannmüller 2013].

Tricuspid valve replacement (TVR) is one of the treatment strategies for TR. Previous studies indicated that TVR is associated with worse early- and long-term outcomes than tricuspid valve repair (TVP) [Singh 2006; Iscan 2007; Guenther 2008; Moraca 2009; Bevan 2014; Hwang 2014]. Nevertheless, because of differences in baseline patient characteristics between TVR and TVP and current indications, operation opportunity and perioperative management are different from those 2 to 3 decades ago, several recently published articles have shown that TVR is not related to extremely higher risk in severe TR patients [Guenther 2008; Moraca 2009; Marquis-Gravel 2012; Raikhelkar 2013; Chang 2017]. Conventional median sternotomy has been regarded over the past decade as the standard approach for TVR for reoperations. Because of perioperative complications, such as severe bleeding, myocardial or lung tissue injury, and even cardiac rupture, patients undergoing reoperation on the TV 

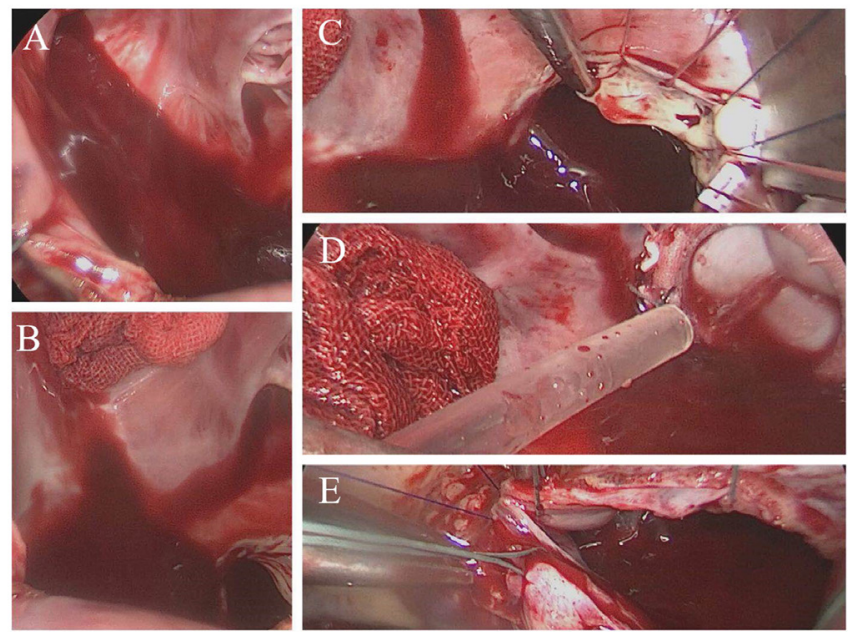

Figure 2. Endoscopic view and technical detail. A, Due to poor drainage of the internal jugular vein cannulations, massive blood flow into the surgical field from the orifice of the superior vena cava occurred. $B$, After inserting the pledget into the superior vena cava, blood from the superior vena cava was significantly reduced, which cleared up the surgical field. C, The entire tricuspid valve is preserved, and the leaflets are folded into the annulus using interrupted mattress with gasket sutures. D, The result of the water flooding experiment. E, Pericardial conglutination is severe, and the incisions of the pericardium and atrium are closed together.

via median sternotomy may have a mortality risk as high as $5 \%-26 \%$ within 30 days after surgery [Iscan 2007; Tokunaga 2008; Mangoni 2001; Maleszka 2004].

The thoracoscopic technique is associated with less trauma, few complications, mild postoperative pain, and quick recovery after surgery. Since 2012, we have utilized uni-port total thoracoscopic technique for TVR in reoperation cases. This study aimed to compare the perioperative and follow-up data of patients who underwent redo TVR via the median sternotomy or thoracoscopic approach and to evaluate the feasibility, safety, and surgical outcomes.

\section{MATERIALS AND METHODS}

Patients: We retrospectively analyzed the records of patients who underwent TVR in our hospital from April 2012 to September 2019. These patients had TR caused by the previous left-sided valve replacement. Written informed consent for publication of clinical details and clinical images were obtained from each patient. The study design was approved by our institutional ethics committee. A total of 49 eligible patients were included in this study. Patients who needed the concomitant left-sided valvular procedure and those with severe respiratory diseases or coronary atherosclerotic heart disease were excluded. Also, patients who performed redo tricuspid valvuloplasty weren't included in this study. In our study population, 26 patients underwent TVR via uni-port total thoracoscopic surgery (study group), whereas 23 patients underwent TVR via median sternotomy
Postoperative pain was evaluated by NRS and FPS-R

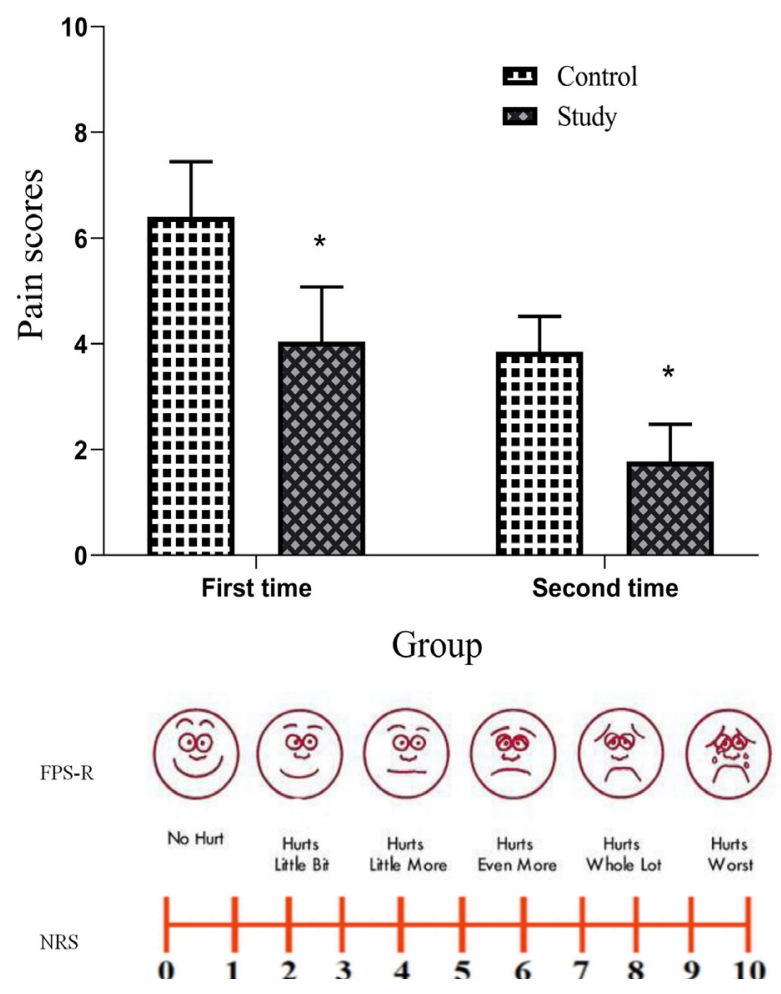

Figure 3. Postoperative pain was evaluated by NRS and FPS-R. No hurt (0 scores), Hurts little bit (1-2 scores), Hurts little more (3-4 scores), Hurts even more (5-6 scores), Hurts whole lot (7-8 scores), Hurts worst (9-10 scores).

(control group). In the study group, the interval between the first and second operations was $16.2 \pm 5.2$ years. With respect to the prior operations, 13, 3, 9, and 1 patients underwent mitral valve replacement (MVR), MVR with concomitant TVP, mitral and aortic valve replacement (DVR), and DVR with concomitant TVP, respectively. In the control group, the interval between the first and second operations was $16.4 \pm 7.3$ years. With respect to prior operations, 12,2 , 8 , and 1 patients underwent MVR, MVR with concomitant TVP, DVR, and DVR with concomitant TVP, respectively. Varying degrees of clinical manifestations were observed among all patients, including palpitations after exercise in 39 patients $(79.6 \%, 39 / 49)$, anhelation in $35(71.4 \%, 35 / 49)$, abdominal distention and sicchasia in 10 (40.8\%, 20/49), bilateral lower extremity and facial edema in $35(71.4 \%$, $35 / 49)$, fatigue in $28(57.1 \%, 28 / 49)$, hydrothorax or ascites in $10(25.6 \%, 10 / 49)$, congestive liver failure or hepatic insufficiency in $12(24.5 \%, 12 / 49)$, and oliguria or renal dysfunction in $10(20.4 \%, 10 / 49)$. The clinical characteristics of the 41 patients are listed in Table 1.

Operative method: Total thoracoscopic TVR - The patients were placed in the supine position with the right 
Table 3. Postoperative data

\begin{tabular}{lccc}
\hline & Study group & Control group & $P$ \\
\hline Time of CPB (min) & $126.0 \pm 31.9$ & $82.1 \pm 38.8$ & $<.001 *$ \\
Operative time (min) & $225.0 \pm 80.8$ & $205.6 \pm 89.7$ & .428 \\
Intraoperative blood loss $(\mathrm{mL})$ & $215.4 \pm 91.6$ & $547.8 \pm 159.2$ & $<.001 *$ \\
Postoperative mechanical ventilation time $(\mathrm{h})$ & $24.0 \pm 15.6$ & $31.1 \pm 24.7$ & $.045^{*}$ \\
Length of ICU $(\mathrm{h})$ & $39.1 \pm 22.2$ & $14.7 \pm 6.4$ & $.002 *$ \\
Postoperative hospital stay (days) & $8.5 \pm 2.7$ & $350.0 \pm 175.2$ & $.001 *$ \\
Thoracic drainage within 24 h after operation $(\mathrm{mL})$ & $235.4 \pm 115.5$ & 14 & $.001 *$ \\
Number of patients receiving blood transfusion & 7 & $.017 *$ & \\
\hline
\end{tabular}

$* P<.05$ versus the controls; CPB, cardiopulmonary bypass; ICU, intensive care unit

Table 4. Early postoperative complications and mortality

\begin{tabular}{|c|c|c|c|c|c|}
\hline Malignant arrhythmia & 0 & 0 & 3 & 13.0 & .096 \\
\hline Renal dysfunction & 1 & 3.8 & 6 & 26.1 & $.033 *$ \\
\hline Hydrothorax & 2 & 7.7 & 7 & 30.4 & $.045 *$ \\
\hline Severe pulmonary infection & 2 & 7.7 & 7 & 30.4 & $.045^{*}$ \\
\hline$I I{ }^{\circ} \mathrm{AVB}$ & 1 & 3.8 & 0 & 0 & .531 \\
\hline MODS & 0 & 0 & 2 & 8.7 & .215 \\
\hline Incisional infection & 0 & 0 & 4 & 17.4 & $.042 *$ \\
\hline Total complications & 6 & 23.1 & 13 & 56.5 & $.017 *$ \\
\hline
\end{tabular}

$* P<.05$ versus the controls

III ${ }^{\circ} \mathrm{AVB}$, three-degree atrioventricular block; LCOS, low cardiac output syndrome;

MODS, multiple organ dysfunction syndrome

shoulder elevated at $30^{\circ}$ and were placed under single-lung ventilation. Electrode slices were placed in every patient for external defibrillation if necessary. A $4 \sim 5 \mathrm{~cm}$ curved skin incision (main working port) was made on the right anterior axillary line. After dissecting a space beneath the pectoralis major muscle, a thoracotomy incision was made in the fourth intercostal space, and a soft-tissue retractor was used to distract the tissue. A $1 \mathrm{~cm}$ incision (auxiliary incision) was made in the third intercostal space at the level of the anterior axillary line. A thoracoscope was inserted through a $1 \mathrm{~cm}$ incision (camera incision) located in the fifth intercostal space on the midaxillary line (Figure 1). A vertical incision in the right inguinal space was made, and the femoral artery and vein were dissected to establish peripheral cardiopulmonary bypass (CPB). We used the Seldinger technique for internal jugular vein cannulation. Femoral-jugular CPB was performed, during beating-heart surgery. Anterior to the phrenic nerves, the pericardium and right atrium were opened together without dissecting the pericardial conglutination. To expose the tricuspid valve (TV), atrium and pericardium were suspended, and an atrial retractor was used if TV exposure was unsatisfactory. A right atrium vent was inserted on the coronary sinus ostium through the auxiliary incision. Valve sizing was performed as usual, and a bioprosthesis was used in all patients. The entire TV was preserved, and we used pledgeted 2-0 braided polyester sutures to fold the leaflets into the annulus using 12 16 interrupted mattress with gasket sutures. This technique is shown in Figure 2C. The prosthetic valve was fixed in place, and all sutures were tied down with the aid of a knot pusher. A water flooding experiment was performed to test the function of bioprosthesis well (Figure 2D). Transesophageal echocardiography was performed to confirm the surgical outcome. 
Table 5. Surgical outcomes

\begin{tabular}{lcc}
\hline & Study group $(\mathrm{N}=26)$ & Control group $(\mathrm{N}=23)$ \\
\hline Cardiac function classification (NYHA) & & \\
I & $18(69.2 \%)$ & $10(52.6 \%)$ \\
II & $8(30.8 \%)$ & $7(42.1 \%)$ \\
III & 0 & $1(5.3 \%)$ \\
Recurrent TR & $3(11.5 \%)$ & .486 \\
++ & 0 & $2(1.1 \%)$ \\
+++ & $.245)$ \\
\hline
\end{tabular}

NYHA, New York Heart Association; TR, tricuspid regurgitation

The incisions of the pericardium and atrium were closed together in double layers (Figure 2E). The internal jugular and femoral vein cannulae were withdrawn at the end of the $\mathrm{CPB}$, and the femoral artery cannula was withdrawn after the reversal with protamine. A chest tube was introduced through the observational incision, and all incisions were sutured closed in a layer-by-layer fashion.

TVR via median sternotomy - In the control group, the median sternotomy was performed, and pericardial conglutination was dissected carefully. Cannulations of the aorta, superior vena cava, and inferior vena cava were applied in all patients, and peripheral CPB was performed during the beating-heart surgery. The right atrium was opened, and whole or partial leaflets were removed. After valve sizing, interrupted mattress with gasket sutures was placed to fix the bioprosthesis in the annulus. The incision of the atrium was closed, the cannulae were withdrawn at the end of the $\mathrm{CPB}$, and the midsternal incision was closed in a layer-by-layer fashion.

Statistical analysis: Enumeration data are expressed as mean \pm standard deviation, and measurement data are expressed as frequencies and percentages. For comparisons, the t-test, Chi-square test, or Fisher's exact test was used. A $P$-value $<.05$ was considered statistically significant. SPSS software (Version 23.0: SPSS Inc., Chicago, IL) was used for statistical analysis.

\section{RESULTS}

Baseline data: There were no statistically significant differences with respect to age, sex, body mass index, or presence of comorbidities, such as hypertension, diabetes, or atrial fibrillation, and the interval between the two operations $(P>.05)$. Hydrothorax, ascites, and liver or kidney dysfunction in both groups were not statistically significant $(P>.05)$. The New York Heart Association classification was used to evaluate the patients' conditions, and there were no significant differences between the groups $(P>.05)$. The clinical characteristics of the patients are shown in Table 1. Preoperative echocardiography was performed for all patients as usual, and the results of these echocardiograms are summarized in Table 2. There were no statistically significant differences in the left ventricular ejection fractions, pulmonary artery pressures, severities of tricuspid regurgitation, or internal diameters of the left atrium, right atrium, and right ventricle.

Comparison of postoperative data: The time of the CPB in the study group was significantly longer than that in the control group $(126.0 \pm 31.9$ min versus $82.1 \pm 38.8 \mathrm{~min}, P<$ $.05)$, but the operative time in the study $(225.0 \pm 80.8 \mathrm{~min})$ and control $(205.6 \pm 89.7 \mathrm{~min})$ groups was not significantly different $(P>.05)$. The following parameters represent the findings, when comparing the study group with the control group in each case: intraoperative blood loss $(215.4 \pm 91.6$ $\mathrm{mL}$ versus $547.8 \pm 159.2 \mathrm{~mL}$ ), postoperative mechanical ventilation time $(24.0 \pm 15.6 \mathrm{~h}$ versus $31.1 \pm 24.7 \mathrm{~h})$, length of intensive care unit (ICU) stay $(39.1 \pm 22.2 \mathrm{~h}$ versus $81.7 \pm 58.0$ h), first postoperative day thoracic drainage $(235.4 \pm 115.5$ $\mathrm{mL}$ versus $350.0 \pm 175.2 \mathrm{~mL}$ ), and postoperative hospital stay $(8.5 \pm 2.7$ days versus $14.7 \pm 6.4$ days $)$. These parameters in the study group were significantly different than those in the control group $(P<.05)$. Compared with the control group, the number of patients receiving blood transfusion was lower in the study group (7/26 versus $14 / 23)$, and the difference was statistically significant $(P<.05)$. The results of the postoperative data are shown in Table 3.

Comparison of postoperative pain: Postoperative pain was evaluated by Numerical Rating Scale (NRS) and Faces Pain Scale Revised (FPS-R). We performed the first pain score when patients transferred to the general wards; we performed pain score for the second time on discharge day. For the first time, the mean values of pain score in the study group were significantly lower than in the control group (4.0 \pm 1.0 versus $6.4 \pm 1.0, P<.05$ ). The second pain score was evaluated on discharge day; the mean values of pain score were $1.8 \pm 0.7$ in the study group and $3.8 \pm 0.7$ in the control group, and the difference was statistically significant $(P<.05)$. When patients transferred to the general wards, the number of patients who used opioids (4/26 versus $9 / 20$ ) and sleeping pills ( $3 / 26$ versus $8 / 20)$ in the study group was lower than those in the control group, and the difference was statistically significant $(P<.05)$. The results of postoperative pain are shown in Figures 3 and 4. 
The use of opioids and sleeping pills in common wards
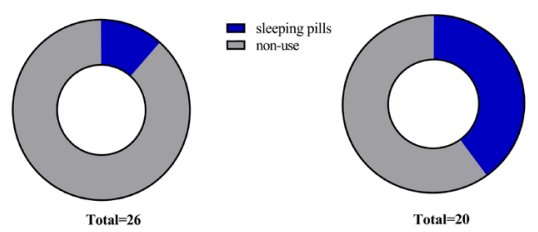

是 slecping pills

stduy group
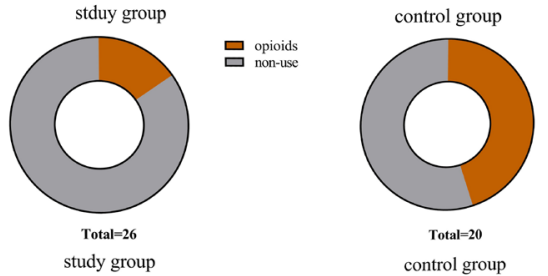

口 opioids

Figure 4. The use of opioids and sleeping pills in common wards. The number of patients who needed opioids and sleeping pills was calculated. Compared with the control group, the patients in the study group needed fewer medications for relieving pain and sleeping. (Opioids: 4/26 versus 9/20); (Sleeping pills:3/26 versus 8/20). Opioids: tramadol, morphine, pethidine, dezocine Sleeping pills: diazepam, oxazepam, estazolam, alprazolam, zolpidem, zopiclone.

Comparison of early postoperative complications and mortality: We compared the incidence of early complications and mortality between the study and control groups. (Table 4) The number of patients with total postoperative complications in the study group was significantly less than in the control group $(P<.05)$. Isolated complication rates were compared separately. The number of patients with renal dysfunction, liver dysfunction, severe pulmonary infection, hydrothorax and incisional infection in the study group was significantly lower than that in the control group $(P<.05)$. However, there was no significant difference between the number of patients with malignant arrhythmia, low cardiac output syndrome (LCOS), three-degree atrioventricular block (III $\left.{ }^{\circ} \mathrm{AVB}\right)$ and multiple organ dysfunction syndrome (MODS) in the two groups $(P>.05)$. Although no patient deaths occurred in the study group and three patients died in the control group in the perioperative period, there were no statistically significant differences in mortality between the two groups $(P>.05)$.

Surgical outcomes and follow-up status: All procedures were completed and no patient required reoperation, due to excessive bleeding or severe complications. No intraoperative deaths occurred in either group. In the study group, there were no conversions to open surgery. A bioprosthesis (27\#$31 \#)$ was used in all patients. Echocardiograms showed the excellent function of the bioprostheses with no evidence of perivalvular leakages. Discharged patients were followed up for three to 36 months postoperatively. One patient was lost to follow up; thus, the overall follow-up rate was $97.83 \%$ (45/46). Excluding one patient in the control group who died of meningitis associated with an epidemic at eight months after discharge, the remaining patients were all alive, during

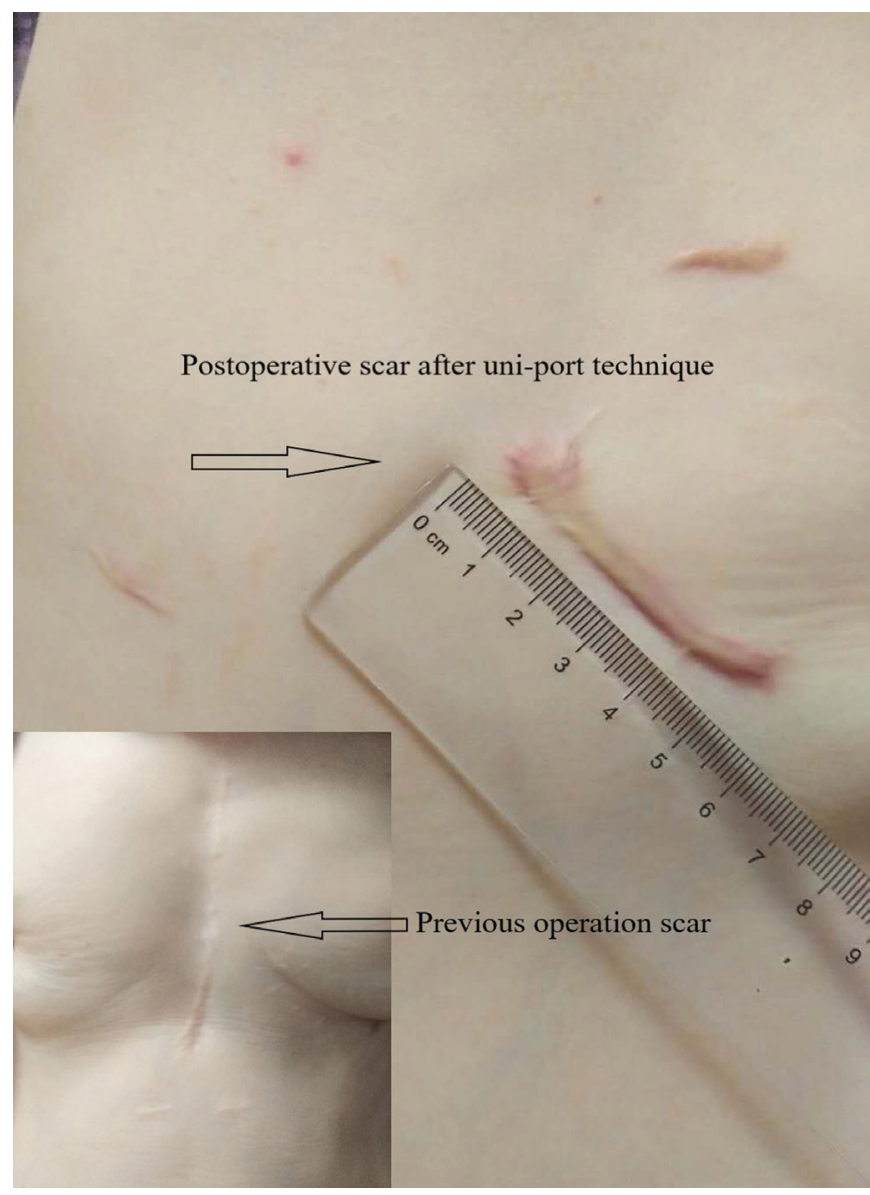

Figure 5. Cosmetic result 1 year after the 'uni-port' thoracoscopic TVR This is a woman's scar 1 year postoperatively, and a small scar of $5 \mathrm{~cm}$ is visible in the right anterior axillary line. A previous operative scar of approximately $25 \mathrm{~cm}$ can be seen in the mid sternal line.

the follow-up period. In the study group, 18 patients were in New York Heart Association (NYHA) class I, and eight patients were in NYHA class II. Recurrent TV regurgitation (++) occurred in three patients. In the control group, 10 patients were in NYHA class I, seven patients were in NYHA class II, and one patient was in NYHA class III. Recurrent TVR $(++)$ occurred in four patients and recurrent TV regurgitation (+++) occurred in two patients. As a group, the patients' cardiac function and activity endurance significantly improved, and the surgical outcome in the study group was no inferior to that of the control group $(P>.05)$. The abovementioned data are shown in Table 5 .

\section{DISCUSSION}

Our study shows that TVR via total thoracoscopic surgery, compared with TVR via median sternotomy, has an obvious advantage in terms of decreased perioperative blood loss and increased speed of postoperative recovery (based on the length of ICU, mechanical ventilation time and hospital stay). 

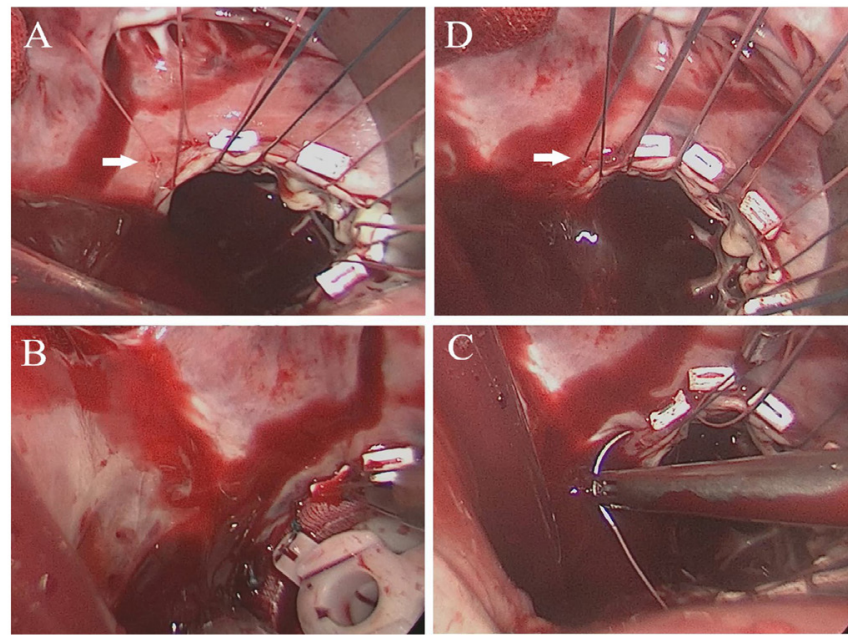

Figure 6. The method of dealing with severe atrioventricular block occurring during the process of TVR. A, Sutures were made too deep at the annulus of septal leaflet, and severe atrioventricular block occurred at the time of knotting. B, All knotted sutures were removed. C, The depth of sutures was adjusted. D, The interrupted mattress with gasket suture was performed once again, and the depth of the suture was shallower in contrast to that of the previous suture.

Total thoracoscopic TVR technically is more challenging and difficult for surgeons, increasing the length of time of $\mathrm{CPB}$. However, there were no significant differences in the operative times between the study and control groups because it was not necessary to dissect the pericardial conglutination, and the postoperative wound closure is easier to perform. By assessing pain scores and the condition of using opioids and sleeping pills, the patients in the study group had less pain and better comfort compared with the control group. Complication rates were calculated, most of the complications (renal dysfunction, liver dysfunction, hydrothorax, and severe pulmonary infection) and total complications in the study group significantly were lower than those in the control group. Follow-up evaluations through clinical or telephone visits revealed excellent surgical outcomes in both groups. Total thoracoscopic TVR is not inferior to traditional surgery concerning cardiac function and recurrence of tricuspid valve regurgitation.

For the patients who enrolled in our study, TVR is the better choice. We believe that submitral structures are damaged after mitral valve replacement, and this damage inevitably affects the heart fiber skeleton that can cause the mechanical balance of the heart fiber skeleton to change. Gradually, the fiber skeleton is displaced, unbalanced, and affected by the TV apparatus [Emilsson 2005]. This mechanical change of the TV and heart fiber skeleton is irreversible; even with TVP, most patients still will have severe tricuspid valve regurgitation after 5-7 years. Furthermore, all patients had obvious annular dilation and crispation of the valve with or without calcifications. Performing TVP should be weighed carefully, due to the high risk of broken or loose sutures and tricuspid annular laceration, especially for the 6 patients who previously had undergone TVP. In a word, tricuspid valve replacement is undertaken when valve repair technically is not feasible or predictably durable. In many patients with secondary TR with marked right ventricular remodeling, leaflet tethering, or stenosis cases, valve replacement can be considered as a primary treatment strategy [El-Eshmawi 2018; Chang 2017]. In contrast to the mechanical prosthetic valve, bioprosthetic valves have more advantages for the opening area and thrombus formation rate. Furthermore, with the development of interventional valve technology, we believe the bioprosthetic valve failure can be resolved in the long term through more minimally invasive interventional techniques. Besides, when the delayed severe atrioventricular block occurs, we can place a pacing lead through the opening of bioprosthesis. These advantages made us inclined to choose a bioprosthesis for TVR.

We have some experience in performing total thoracoscopic TVR. We chose to make an incision in the 4th intercostal space on the right anterior axillary line for the main working port and then chose to make two incisions in the $3 \mathrm{rd}$ and 5th intercostal spaces as the camera and auxiliary incisions, respectively (Figure 1). These incisions ensured improved views of the operative field and flexibility of the operative manipulations, simultaneously; they also yield better cosmetic effects (a postoperative scar is shown in Figure 5. To prevent unnecessary bleeding, injury, and the risk of atrial rupture, we incised the pericardium and right atrium together without dissecting the pericardial conglutination, which is the most time-consuming part of the classical approach. In some cases, poor drainage of the femoral vein and internal jugular vein cannulations made the surgical field unclear, and we could not block the vena cava by taping the superior and inferior vena cava because of atri-pericardium incision. Therefore, pledget or Foley's catheter was placed in the orifices of the superior or inferior vena cava (Figure 2A and $2 \mathrm{~B}$ ). Use of vena cava negative pressure assist drainage technique can ensure satisfactory vena cava drainage, and a right atrium vent was inserted on the coronary sinus ostium. This process ensures a clearer surgical field. The entire TV was preserved, and the leaflets were folded into the annulus instead of removing them; therefore, we were able to maintain the integrity of the chordae and papillary muscles. Given the irregular geometry of the right ventricle and the thinner right ventricular wall compared with the left side of the heart, the chordae and papillary muscles served as significant mechanical fulcra to stabilize the right ventricular structure and minimize ventricular enlargement. Besides, these structures are important to enhance the contractile efficiency of the right ventricle by maintaining the concentric contraction of the right ventricle. Also, preserving the TV can reduce the risk of bleeding, myocardial injury, and even right ventricular rupture when the leaflets and the surrounding tissue are removed. It is also technically important to prevent and handle the occurrence of an atrioventricular block, during the process of TVR. Other than TVP, the process of TVR inevitably needs to suture at the annulus of the septal leaflet. We consider that the sutures, when performed at the root of valve, keep off the Koch triangle to prevent damage to the $\mathrm{AV}$ node. If the annulus of the septal leaflet is sutured too 
deep, the heart rate will slow down at the time of knotting, and a severe atrioventricular block can be observed on the ECG monitor. Under this condition, the knotted suture is removed, the suturing is reperformed, and the depth of the suture is adjusted (Figure 6). Conversely, interrupted mattress sutures are made on the valve leaflet with re-knotting.

\section{CONCLUSIONS}

In conclusion, uni-port total thoracoscopic surgery is a safe, feasible, and promising approach for TVR. Furthermore, it has the advantages of better cosmetic effects, decreased bleeding, few complications, mild postoperative pain, quick recovery and improved cardiac function and quality of life. We suggest that uni-port total thoracoscopic TVR can be considered as a primary treatment strategy for patients with severe TR after previous left-sided valve procedure.

\section{REFERENCES}

Anyanwu AC, Adams DH. 2010. Functional tricuspid regurgitation in mitral valve disease: epidemiology and prognostic implications. Semin Thorac Cardiovasc Surg 22(1):69-75.

Bellavia D, Pentiricci S, Senni M, Gavazzi A. 2014. Update on tricuspid regurgitation. G Ital Cardiol (Rome). 15(7-8):418-429.

Benedetto U, Melina G, Angeloni E, et al. 2012. Prophylactic tricuspid annuloplasty in patients with dilated tricuspid annulus undergoing mitral valve surgery. J Thorac Cardiovasc Surg 143(3):632-638.15.

Bevan PJW, Haydock DA, Kang N. 2014. Long-term survival after isolated tricuspid valve replacement. Heart Lung Circ 23(8):697-702.

Chang HW, Jeong DS, Cho YH, et al. 2017. Tricuspid Valve Replacement vs. Repair in Severe Tricuspid Regurgitation. Circ J. 81(3):330-338.

Chikwe J, Itagaki S, Anyanwu A, Adams DH. 2015. Impact of Concomitant Tricuspid Annuloplasty on Tricuspid Regurgitation, Right Ventricular Function, and Pulmonary Artery Hypertension After Repair of Mitral Valve Prolapse. J Am Coll Cardiol 65(18):1931-1938.

Dreyfus GD, Corbi PJ, Chan KMJ, Bahrami T. 2005. Secondary tricuspid regurgitation or dilatation: which should be the criteria for surgical repair? Ann Thorac Surg. 79(1):127-132.

El-Eshmawi A, Pandis D, Adams DH, Tang GH. 2018. Tricuspid valve surgery: repair and replacement. Minerva Cardioangiol. 66(6):700-712.

Emilsson K, Egerlid R, Nygren B. 2005. Tricuspid annulus motion and mitral annulus motion: Anatomical intimacy causing a good correlation? Exp Clin Cardiol 10(2):111-115.

Gosev I, Yammine M, McGurk S, et al. 2016. Should Moderate-toSevere Tricuspid Regurgitation be Repaired During Reoperative LeftSided Valve Procedures? Semin Thorac Cardiovasc Surg 28(1):38-45.

Guenther T, Noebauer C, Mazzitelli D, Busch R, Tassani-Prell P, Lange R. 2008. Tricuspid valve surgery: a thirty-year assessment of early and late outcome. Eur J Cardiothorac Surg 34(2):402-409.

He J, Shen Z, Yu Y, et al. 2012. Criteria for determining the need for surgical treatment of tricuspid regurgitation during mitral valve replacement. J Cardiothorac Surg 7:27-27.

Hwang HY, Kim K-H, Kim K-B, Ahn H. 2014. Treatment for severe functional tricuspid regurgitation: annuloplasty versus valve replacement. Eur J Cardiothorac Surg 46(2):e21-e27.

Iscan ZH, Vural KM, Bahar I, Mavioglu L, Saritas A. 2007. What to expect after tricuspid valve replacement? Long-term results. Eur J Cardiothorac Surg 32(2):296-300.

Jamieson WR, Edwards FH, Schwartz M, Bero JW, Clark RE, Grover FL. 1999. Risk stratification for cardiac valve replacement. National Cardiac Surgery Database. Database Committee of The Society of Thoracic Surgeons. Ann Thorac Surg 67(4):943-951.

Lange R, Piazza N, Günther T. 2017. Tricuspid valve regurgitation : Indications and operative techniques. Herz. 42(7):653-661.

Maleszka A, Kleikamp G, Koerfer R. 2004. Tricuspid valve replacement: clinical long-term results for acquired isolated tricuspid valve regurgitation. J Heart Valve Dis. 13(6):957-961.

Mangoni AA, DiSalvo TG, Vlahakes GJ, Polanczyk CA, Fifer MA. 2001. Outcome following isolated tricuspid valve replacement. Eur J Cardiothorac Surg. 19(1):68-73.

Marquis-Gravel G, Bouchard D, Perrault LP, et al. 2012. Retrospective cohort analysis of 926 tricuspid valve surgeries: clinical and hemodynamic outcomes with propensity score analysis. Am Heart J. 163(5):851858.e851.

McCarthy PM, Bhudia SK, Rajeswaran J, et al. 2004. Tricuspid valve repair: durability and risk factors for failure. J Thorac Cardiovasc Surg 127(3):674-685

Moraca RJ, Moon MR, Lawton JS, et al. 2009. Outcomes of tricuspid valve repair and replacement: a propensity analysis. Ann Thorac Surg 87(1):83-89.

Nishimura RA, Otto CM, Bonow RO, et al. 2014. 2014 AHA/ACC guideline for the management of patients with valvular heart disease: a report of the American College of Cardiology/American Heart Association Task Force on Practice Guidelines. J Am Coll Cardiol 63(22):e57-e185.

Pfannmüller B, Moz M, Misfeld M, et al. 2013. Isolated tricuspid valve surgery in patients with previous cardiac surgery. J Thorac Cardiovasc Surg 146(4):841-847.

Pinney SP. 2012. The role of tricuspid valve repair and replacement in right heart failure. Curr Opin Cardiol 27(3):288-295.

Pozzoli A, Lapenna E, Vicentini L, Alfieri O, De Bonis M. 2016. Surgical indication for functional tricuspid regurgitation at initial operation: judging from long term outcomes. Gen Thorac Cardiovasc Surg. 64(9):509-516.

Raikhelkar J, Lin H-M, Neckman D, Afonso A, Scurlock C. 2013. Isolated tricuspid valve surgery: predictors of adverse outcome and survival. Heart Lung Circ 22(3):211-220.

Singh SK, Tang GHL, Maganti MD, et al. 2006. Midterm outcomes of tricuspid valve repair versus replacement for organic tricuspid disease. Ann Thorac Surg 82(5):1735-1741.

Tokunaga S, Masuda M, Shiose A, Tomita Y, Morita S, Tominaga R. 2008. Long-term results of isolated tricuspid valve replacement. Asian Cardiovasc Thorac Ann. 16(1):25-28.

Tornos Mas P, Rodríguez-Palomares JF, Antunes MJ. 2015. Secondary tricuspid valve regurgitation: a forgotten entity. Heart. 101(22):1840-1848.

Van de Veire NR, Braun J, Delgado V, et al. 2011. Tricuspid annuloplasty prevents right ventricular dilatation and progression of tricuspid regurgitation in patients with tricuspid annular dilatation undergoing mitral valve repair. J Thorac Cardiovasc Surg 141(6):1431-1439. 